International Journal of Distributed and Parallel Systems (IJDPS) Vol.2, No.2, March 2011

\title{
A Dynamic MAC Protocol with Adaptive Power Control for WCDMA Networks
}

\author{
S.P.V.Subba Rao ${ }^{1}$ Dr.S. Venkata Chalam ${ }^{2}$ Dr.D.Sreenivasa Rao ${ }^{3}$ \\ ${ }^{1}$ Department of Electronics and Communication Engineering, Sreenidhi Institute of \\ Science and Technology, Hyderabad, India. spvsr2007egmail.com \\ ${ }^{2}$ Department of Electronics and Communication Engineering, \\ ACE Engineering College, Hyderabad, India. svchalam2003@yahoo.com \\ ${ }^{3}$ Department of Electronics and Communication Engineering, \\ Jawaharlal Nehru Technological University, Hyderabad, India. dsraoece@gmail.com
}

\begin{abstract}
In Wideband code-division multiple-access (WCDMA) systems, all users share the same time and frequency resources and may lead to near far problem, where power control forms an effective solution. A power control algorithm aims to reduce transmission power and interference level, and to maximize system capacity. In this paper, we propose an adaptive power control mechanism for multimedia traffic in WCDMA networks. The proposed algorithm uses two most recent Transmit Power Control (TPC) commands to compute the Adaptive Factor (AF) based on a predefined Adaptive Control Factor (ACF). We introduce another parameter, Power Determining Factor (PDF) based on the data traffic rate to determine the power. Based on this parameter, the power is increased or decreased. Depending on the traffic rate, the PDF factor is updated i.e., if the observed traffic rate is high, then it will increase the parameter and subsequently increases the power and if it is low, then the parameter will be decreased and correspondingly the power also. By simulation results, we show that the proposed power control algorithm reduces the power consumption of multimedia traffic.
\end{abstract}

\section{KEY WORDS}

Wideband code-division multiple-access (WCDMA), adaptive power control, Transmit Power Control

\section{INTRODUCTION}

\subsection{WCDMA}

Wideband code-division multiple-access (WCDMA) [1] forms the basis of the air interface in the $3^{\text {rd }}$ generation cellular mobile communications. It has higher speeds and it supports more users as it utilizes the direct-sequence spread spectrum method of asynchronous code division multiple accesses. WCDMA is a wide band spread- spectrum channel access method and is a type of $3 \mathrm{G}$ cellular network. The technology behind the 3G UMTS [2] standard is WCDMA and is allied with the 2G GSM standard. Here in WCDMA, each signal transmitted in the air interface is presented as a wideband noise to the desired signal and so is an interference limited system. If there are a large number of users, then the mutual interference between the connections degrades the QoS for the new user as well for the ongoing connections [3]. WCDMA is adaptable to distribute multimedia traffic [4]. 
International Journal of Distributed and Parallel Systems (IJDPS) Vol.2, No.2, March 2011

\subsection{POWER CONTROL}

There are different channels in the CDMA system and the minimum Signal to Interference Ratio (SIR) of each channel is maximized by the power control mechanisms. So in order to increase the capacity of CDMA system in terms of the number of concurrent calls in the commonly used bandwidth [5], we must use an efficient power control design. The power control reduces the mutual interference between the users and near-far fading effects and it also maintain acceptable connections in wireless communications systems [6].As it reduces the interference, the cell capacity improves and thus increase the battery life by using a minimum transmitter power. The main characteristics of power control are compensation of fading channels and changes in the transmitted powers of interfering users. Power control is also known as the Transmit Power Control (TPC) [11] since each user changes their access to the resources by setting its transmitting power to the changing channel and interference conditions. TPC is a considerable design problem in CDMA systems.

The power control in WCDMA is SIR based [1], [5]. In WCDMA, there may exist near far problem, since all users share the same time and frequency resources. Power control forms an effective solution to this problem. The power control algorithms maximize the system capacity and it reduces the transmission power and interference level [7]. In order to maximize the total capacity in the cell, the power control in uplink must make signal powers from different users nearly equal. By making the transmission power level received from all terminals as good as possible at the home cell for the same QoS, the uplink power control lessens the near-far problem. In downlink, power control keeps the signal at minimal required level to reduce the interference to users in other cells and the signals transmitted are mutually orthogonal [10] and that is why they do not interfere with each other.

\section{Types of Power Control}

There are three types of power control in WCDMA,

- The open loop power control: is for initial power setting of mobile station across the air in terface.

- Fast closed loop power control: can mitigate fast fading rate up to $1.5 \mathrm{kbps}$ on uplink a $\mathrm{n} \mathrm{d}$ downlink. It uses a fixed quality target set in mobile station or base station.

- Outer loop power control: compensates changes in environment. It adjusts the SIR target to achieve the required FER/BER/BLER. It depends on MS speed available, and keeps multi path diversity [3].

Power of every transmitter is adjusted to the level required to meet the requested QoS. Due to dynamic variation of the radio channel, the determination of the transmitted power is a difficult task. The received power should be at an acceptable level [10], irrespective of the radio environments.

\subsection{PROBLEM IDENTIFICATION AND PROPOSED SOLUTION}

In our previous work [14], traffic is classified as Real time (RT) and Non Real time (NRT) and prioritized as High and low, respectively. For the high priority data traffic, a node can be in one of two modes: low data traffic (LDT) or high data traffic (HDT). A node is in HDT only when it receives a High Contention (HC) Message (HCM) from a two-hop neighbor within the last T period. Otherwise, the node is in LDT. A node sends an HCM when it experiences high contention due to high data traffic. Each node calculates the data traffic (DT) to calculate the 
International Journal of Distributed and Parallel Systems (IJDPS) Vol.2, No.2, March 2011

contention. If DT is more than a threshold value $\mathrm{DT}_{\mathrm{th}}$, then the nodes will send a HCM. In this paper, we propose an adaptive power control mechanism for multimedia traffic in WCDMA networks.

In addition to the maximum and minimum values [13] of the step sizes which are common parameters for all adaptive power control techniques, an Adaptive Control Factor (ACF), is also involved in this technique. The power control step size is adapted by multiplying a factor called Adaptive Factor (AF) [12] with the fixed step size. This factor will be updated according to received TPC commands.

In the proposed algorithm, we are introducing another parameter, Power Determining Factor (PDF) based on the data traffic rate, to determine the power. Based on this parameter, we are determining whether the power is increasing or decreasing. Depending on the traffic rate, the PDF factor is updated i.e., If the received message is HCM, then it will increase the parameter and subsequently increases the power and if the message is Low contention (LC), then the parameter will be decreased and correspondingly the power also.

The paper is organized as follows: Section 2 presents the existing works on power control. Section 3 describes the Proposed Adaptive Power Control Technique. Section 4 gives the Simulation Results and performance comparison of protocol. Section 5 concludes the paper.

\section{Existing Works on PoWer Control}

S. A. El-Dolil et al. [3] have proposed a model to investigate the performance of multi-cell admission control when heterogeneous traffic was considered. Tradeoffs between the dropping and blocking probabilities have been discussed. As dropping an ongoing call was more annoying than blocking a new one, dropping probability could be lowered without much increase in blocking probability. Both homogenous and heterogeneous traffic were considered; and concluded that more capacity gain is achieved under heterogeneous (hot around) traffic distribution. But the results showed that high bit rate services suffered from both higher blocking and dropping probabilities, so it was assumed that these services were with limited coverage.

N. Mohan [4] has proposed a new CAC algorithm with power control for multiple services like voice, video and data for multiclass users. It determined the optimum set of admissible users with the optimum transmitting power level, so as to minimize the interference level and call rejection rate. In addition to that, an adaptive scheduling scheme to allocate optimum rate for each traffic queue was also proposed to minimize the scheduling delay.

Markus Laner et al. [7] have proposed the uplink OLPC related large-scale measurements, performed on live Iub interfaces. Evaluations of the actual implemented algorithm showed that it converges slowly; the reason being that the QoS was estimated by CRC. As the uncoded Bit Error Ratio (BER) holds information about the QoS, that parameter could be used to increase convergence speed of the OLPC. They presented a statistical model of the control path of the OLPC which takes the uncoded BER information into account.

Rekha Patil and A. Damodaram [9] have proposed, a cross-layer based joint scheduling and power control algorithm with the objective of minimizing the interference level and call rejection rate. For achieving that, the algorithm determined the optimum set of admissible users with suitable transmitting power level. They solved the multiple access problems in the 
International Journal of Distributed and Parallel Systems (IJDPS) Vol.2, No.2, March 2011

distributed power control algorithm, the set of powers that could be used by the scheduled users to satisfy their transmissions were determined.

Rachod Patachaianand, Kumbesan Sandrasegaran [13] has showed that there is a significant correlation between TPC sequences and user mobility. They introduced a new parameter called Consecutive TPC Ratio (CTR), which will be varied by user speeds. A new adaptive power control algorithm was also proposed by them. The new power control algorithm uses CTRs to adjust power control step sizes.

Rachod Patachaianand, Kumbesan Sandrasegaran [12] have first investigated the performance of current power control algorithms and then proposed a new adaptive power control algorithm for UMTS. The proposed algorithm could mitigate received SIR variations when channel fading changed slowly. It was capable of tracking rapid changes in fast fading channels, where other UMTS power control algorithms failed to handle.

\section{Proposed Adaptive PoWer Control Technique}

\subsection{Outer and Inner Loop Power Control}

The aim of a power control procedure is to ensure an adequate SIR for all mobiles in a system by a simple algorithm. The algorithm requires feedback information from the receiver and based on that, the transmitting power is adjusted at transmitter side. The information regarding the feedback is delivered by means of the feedback information. The bandwidth of the feedback is expensive and so the amount of information required by the procedure must be kept minimal.

There are outer and inner loop power controls. The outer-loop power control is performed by Radio Network Controller (RNC). Based on some particular measured parameters such as BLER (Block Error Rate), the outer loop power control adjusts the SIR target. The required BLER depends on radio conditions and service types. According to the conditions and service types, the individual SIR target of each mobile will be set. The requirement of SIR target will be higher if a mobile moves quickly resulting in rapid change in radio channel and vice versa. In addition, different service types require different BLER. The SIR targets of data services are higher than the voice service as the data service requires lower BLER than voice services. The frequency of SIR target updating is $10-100 \mathrm{~Hz}$ [12].

Inner loop power control mechanism adjusts the transmitted power to maintain the received SIR equal to the SIR target at the receiver. In 3GPP specifications, each WCDMA frame of length 10ms consists of 15 time slots and each of which consists of one bit of the power control command called "Transmit Power Control" or TPC.TPC is a command, which increases or decreases the transmitting power. The transmitter will be controlled to increase OR decrease the transmit power, so that the power will always be oscillated even if the received SIR is close to the SIR target. The step size will be increased if same TPC commands are detected or else the updated step is very large, the step size will then be decreased.

The transmit power can be represented as:

$\mathrm{P}(\mathrm{t}+1)=\mathrm{P}(\mathrm{t})+\lambda$. sign $($ SIR target - SIRest $)[\mathrm{dB}]$

Where $\mathrm{P}(\mathrm{t})$ represent the transmit power at time $\mathrm{t}, \lambda$ is the power control step size, SIRtarget, and SIRest are the target and estimated SIR respectively. The term sign is the sign function: $\operatorname{sign}(\mathrm{x})=1$, when $\mathrm{x} \geq 0$, and $\operatorname{sign}(\mathrm{x})=-1$, when $\mathrm{x}<0$.

It can be noted that sign (SIRtarget-SIRest) $=-1$ is equivalent to a TPC power up command which can be represented by bit 0 . From Equation 1, it can be observed that the transmit power 
will be increased or decreased by $\lambda$ on every time slot. The transmitted power will always change even when there is no change in the channel.

\subsection{Adaptive Step Size Estimation}

The power control step size is adapted by multiplying a factor called Adaptive Factor (AF) with the fixed step size. This factor will be updated according to received TPC commands. The proposed algorithm increases the step size i.e. AF when the mobile detects the same sequence of TPC. The same sequence of TPC commands will occur when rapid changes of power are required, such as when the radio channel condition variants rapidly and continuously.

The proposed algorithm uses two most recent TPC commands to compute the AF based on a predefined ACF. The ACF is a constant chosen by the networks.

The AF will be updated by the following equation:

$\mathrm{AF}_{\mathrm{u}}(\mathrm{t})=\min \left(\max \left(\mathrm{ACF}+\mathrm{DF}_{\mathrm{u}}(\mathrm{t}) / \mathrm{ACF}, \mathrm{Size}_{\min )}, \operatorname{Size}_{\max }\right)\right.$

Where, Size $\mathrm{min}_{\min }$ and $\mathrm{Size}_{\max }$ is the minimum and maximum step size respectively. The $\mathrm{DF}_{\mathrm{u}}(\mathrm{t})$ is the Dynamic Factor of $\mathrm{u}^{\text {th }}$ user at time $\mathrm{t}$.

In the proposed algorithm, we are introducing another parameter, Power Determining Factor (PDF) based on the data traffic rate to determine the power. Based on this parameter we are determining whether the power is increasing or decreasing. Depending on the traffic rate, the PDF factor is updated.

From our previous work,[14] each node i calculates the traffic rate by using the traditional way to calculate the system capacity for data traffic (DT), which is given by;

$$
D T i=\left[\frac{\alpha 1}{S I R}\right] \times \frac{1}{1+\kappa} \times P \times \frac{1}{\sigma} \times \alpha 2
$$

Where,

$\mathrm{DT}_{\mathrm{i}} \quad=$ Data traffic for $\mathrm{i}^{\text {th }}$ node.

$\alpha 1$ And $\alpha 2=$ the processing gain by spectrum spreading and gain due to sector antenna Respectively.

SIR = Signal to interference ratio

$\kappa=\quad=$ The interference from other nodes

$\mathrm{P}=$ the power control factor

$\sigma \quad=$ The voice/data activity factor.

Every neighbor calculates the traffic to deduce the contention.

Therefore data transfer for $\mathrm{u}^{\text {th }}$ user is given by:

$\mathrm{n}$

$\mathrm{DT}_{\mathrm{u}}(\mathrm{t})=\sum \mathrm{DTi}$

$\mathrm{i}=1$

So $\operatorname{PDF}_{\mathrm{u}}(\mathrm{t})=\mathrm{DT}_{\mathrm{u}}(\mathrm{t})$

From Equation 2, AFu (t) is linearly updated by DTu (t). That is only a few additional complexity is required at the mobiles. 
The DF is updated based on two TPC commands as the following equation:

$\mathrm{DF}_{\mathrm{u}}(\mathrm{t})=\mathrm{DF}_{\mathrm{u}}(\mathrm{t}-1)-\mathrm{abs}\left(\mathrm{TPC}_{\mathrm{u}}(\mathrm{t})-\mathrm{TPC}_{\mathrm{u}}(\mathrm{t}-1)\right)+1$

Where $\operatorname{abs}(\mathrm{x})$ is the absolute value of $\mathrm{x}$.

The transmit power is updated according to the following equation:

$\mathrm{P}_{\mathrm{u}}(\mathrm{t}+1)=\mathrm{P}_{\mathrm{u}}(\mathrm{t})+\mathrm{AF}_{\mathrm{u}}(\mathrm{t}) \cdot \mathrm{PDF}_{\mathrm{u}}(\mathrm{t}) \lambda \cdot \mathrm{TPC}_{\mathrm{u}}(\mathrm{t})$

Where $\mathrm{AFu}(\mathrm{t})$ is the Adaptive Factor of $\mathrm{u}^{\text {th }}$ user at time $\mathrm{t}$, and TPCu(t) is the TPC command of $\mathrm{u}^{\text {th }}$ user at time $t$, corresponding to sign (SIRtarget-SIRest) in Equation 1, and $\operatorname{PDF}_{u}(t)$ is the Power Determining Factor.

If the received message is $\mathrm{HCM}$, then it will increase the parameter and subsequently increases the power and if the message is LC, then the parameter will be decreased and correspondingly the power also.

\section{Simulation RESUlts}

\subsection{Simulation Setup}

In this section, we simulate the proposed dynamic MAC protocol with adaptive power control (DMAC-APC) algorithm for WCDMA cellular networks. The simulation tool used is NS2 [15] which is a general-purpose simulation tool that provides discrete event simulation of user defined networks. In the simulation, mobile nodes move in a 600 meter x 600 meter region for 50 seconds simulation time. All nodes have the same transmission range of 250 meters. The simulation parameters are given in table 1 .

\begin{tabular}{|l|l|}
\hline Area Size & 600 X 600 \\
\hline Number of Cells & 2 \\
\hline Users Per Cell & 20 \\
\hline Slot Duration & $2 \mathrm{~m} \mathrm{sec}$ \\
\hline Radio Range & $250 \mathrm{~m}$ \\
\hline Frame Length & 2 to 8 slots \\
\hline Frame Duration & 1 second \\
\hline CDMA codes & 2 to 5 \\
\hline Simulation Time & 50 sec \\
\hline Routing Protocol & AODV \\
\hline Traffic Source & CBR, VBR \\
\hline Packet Size & 512 bytes \\
\hline MSDU & 2132 \\
\hline $\begin{array}{l}\text { Transmission } \\
\text { Rate }\end{array}$ & $1 \mathrm{Mb}$ \\
\hline No. of Users & $2,4,6,8$ \\
\hline
\end{tabular}

Table I. Simulation Parameters 


\subsection{Performance Metrics}

The performance is mainly evaluated according to the following metrics:

Channel Utilization: It is the ratio of bandwidth received into total available bandwidth for a traffic flow.

Throughput: It is the throughput received successfully, measured in $\mathrm{Mb} / \mathrm{s}$.

Average Energy: It is the average energy consumption of all nodes in sending, receiving and forward operations.

The proposed DMAC-APC is compared with the DMAC [14] protocol without using power control.

The performance results are presented in the next section.

\subsection{Results}

In the simulation experiment, the number of users is varied as 2, 4, 6, 8 and 10 and the channel utilization, throughput and energy consumption are measured.

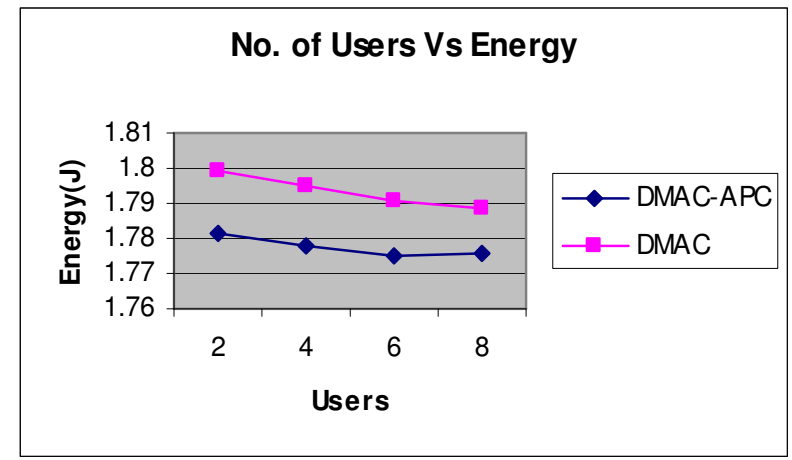

Fig 1: Users Vs Energy

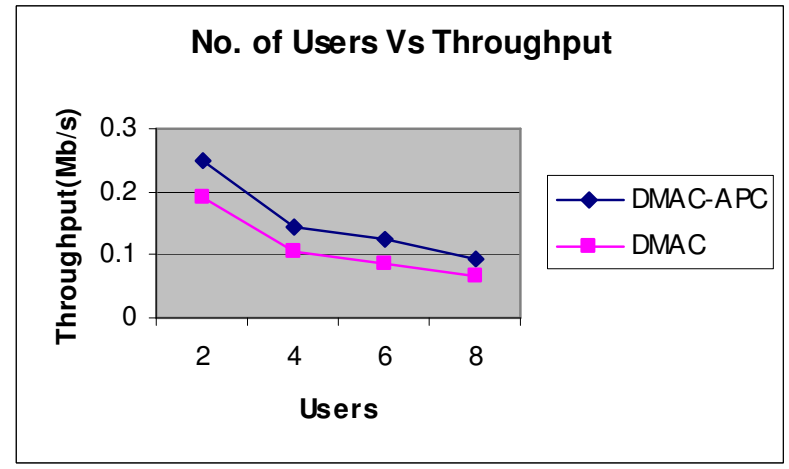

Fig 2: Users Vs Throughput 


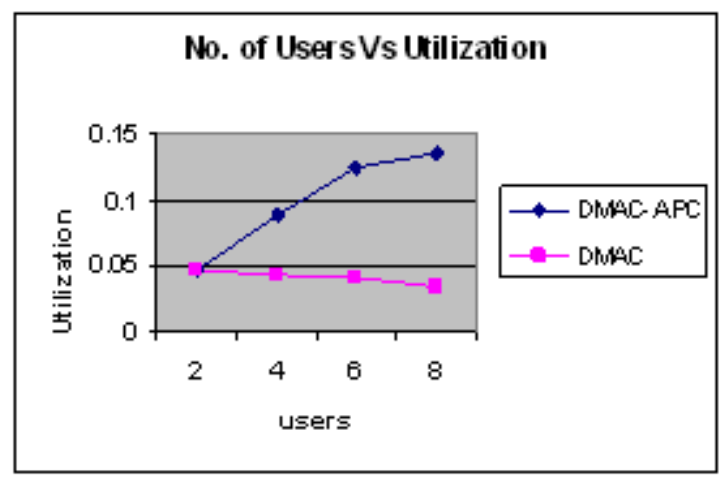

Fig 3: Users Vs Utilization

Figure 1 shows the energy consumption for both DMAC and DMAC-APC. Because of its adaptive power control technique, DMAC-APC has reduced energy consumption when compared with DMAC. The reason is that in DMAC-APC for low data traffic the power is lowered and for the high data traffic the corresponding power is increased. In DMAC protocol the users data is transmitted without power control hence energy consumption is more than DMAC-APC.

Figure 2 shows the throughput obtained with the DMAC-APC and DMAC protocols. From the figure, it can be seen that, the throughput of both protocols are decreased, when the users are increased. But it shows that the throughput is more for DMAC-APC, because in DMAC-APC protocol the power control mechanism adjusts the transmitter power to maintain the required SIR equal to the SIR target value. So the data is transmitted with less BER successfully. Hence the throughput is more.

In DMAC the user data is transmitted without power control and the required SIR is not equal to the SIR target value. Hence the user data is not transmitted successfully. Hence the throughput is less.

It can be seen from Figure.3 DMAC-APC has better channel utilization than the DMAC protocol. In DMAC APC the channel utilization gradually increases when the number of users is increased. The reason for better channel utilization is due to the very adaptive nature of power control that allows the network to offer quality of service for different traffics by efficiently adjusting the power and improving the capacity of the network. In DMAC When data traffic is high, more number of users will contend and hence fair utilization of the channel will be decreased.

\section{CONCLUSION}

In WCDMA systems, all users share the same time and frequency resources and may lead to near far problem, where power control forms an effective solution. The power control algorithm aims to reduce transmission power and interference level, and to maximize system capacity. As it reduces the interference, the cell capacity improves and thus increases the battery life by using a minimum transmitter power. In this paper, we have designed an adaptive power control mechanism for multimedia traffic in WCDMA networks. In addition to the maximum and minimum values for the step sizes which are common parameters for all adaptive power control techniques, an ACF, involved in this technique. The power control step size is adapted by multiplying a factor called AF with the fixed step size. This factor will be 
International Journal of Distributed and Parallel Systems (IJDPS) Vol.2, No.2, March 2011

updated according to received TPC commands. Here we are introducing another parameter, Power Determining Factor (PDF) based on the data traffic rate to determine the power. Based on this parameter we are determining whether the power is increasing or decreasing. Depending on the traffic rate, the PDF factor is updated i.e., if the observed traffic rate is high, then it will increase the parameter and subsequently increases the power and if it is low, then the parameter will be decreased and correspondingly the power also. By simulation results, we have shown that the proposed power control algorithm reduces the power consumption of multimedia traffic.

\section{REFERENCES}

[1] Dejan Drajic, Ericsson d. and V.Popovica "Turbo Coding and Power Control for WCDMA Downlink", in proceedings of wireless personal communications, vol.24, No. 4, pp.259-276, Belgrade, Yugoslavia, 2004.

[2] Cheng Soon Wai and Ooi Wen Hui,"Communication Radio \& Satellite", pp. 2-14, 27 October 2009.

[3] S. A. El-Dolil, A. Y. Al-nahari, M. I. Desouky and F. E. Abd El-samie "Uplink Power Based Admission Control in Multi-cell Wcdma networks with Heterogeneous Traffic" In proceedings of Progress in Electromagnetics Research B, vol. 1, pp. 115-134, Egypt, 2008.

[4] N. Mohan "An Efficient Multiclass Call Admission Control and Adaptive Scheduling for WCDMA Wireless Network" In proceedings of European Journal of Scientific Research, vol.33, No.4, pp.718-727, 2009.

[5] Siamäk Naghian, Matti Rintamäki and Ramin Baghaie "Dynamic Step-Size Power Control in umts", in proceedings of the 13th IEEE International Symposium on Personal, Indoor and Mobile Radio Communications PIMRC ,vol. 4, pp.1606 - 1610, Lissabon, Portugal, September, 2002.

[6] redrik Gunnarsson "Fundamental Limitations of Power Control in WCDMA" 6th Swedish CDMA Workshop, December 4, 2001.

[7] Markus Laner, Philipp Svoboda and Markus Rupp, "Outer-Loop Power Control in a Live UMTS Network: Measurement, Analysis and Improvements" In proceedings of $4^{\text {th }}$ International symposium on Communications, Control and Signal processing, Cyprus, 3-5 March 2010.

[8] Cynara C Wu and Dimitri PBertsekas," Distributed Power Control Algorithms for Wireless Networks" in proceedings of , IEEE Transactions on Vehicular Technology, vol. 50, No. 2, pp.504 - 514, USA, 2001.

[9] Rekha Patil and A. Damodaram,"A Cross-Layer Based Joint Algorithm for Power Control and Scheduling in CDMA Wireless Ad-Hoc Networks", In proceedings of wseas transactions on communications,vol.8,No.1, pp.122-131, January, 2009.

[10] Sukhpreet Kaur Khangura Kiranpreet Kaur and R. S. Uppal, "Power Control Algorithms in Wireless Communication", International Journal of Computer Applications, vol.1, No.12, pp.975 - 8887, 2010.

[11] Abdurazak Mudesir,"Power Control Algorithm in CDMA systems", Guided research, 2004.

[12] Rachod Patachaianand, Kumbesan Sandrasegaran "A New Adaptive Power Control Algorithm for UMTS", WITSP, Wollongong, Australia, pp. 1-6, 2006.

[13] Rachod Patachaianand, Kumbesan Sandrasegaran, “An Adaptive Step Size Power Control With Transmit Power Control Command Aided Mobility Estimation" In proceedings of the fourth IASTED Asian Conference Communication Systems and Networks, Thailand,2007.

[14] S.P.V.Subbarao, S. Venkata Chalam and D.Srinivasa Rao, "A Dynamic MAC Protocol for WCDMA Wireless Multimedia Networks", International Journal on Network Security (IJNS).

[15] Network Simulator, http://www.isi.edu/nsnam/ns 
International Journal of Distributed and Parallel Systems (IJDPS) Vol.2, No.2, March 2011

\section{AUTHORS}

S.P.V.SubbaRao is graduated in B.Tech (Electronics and Communication Engineering) in 2000 from the JNT University, Hyderabad. He did his M.Tech in Communications and signal processing from S.K University, Anantapur during 2005.He is pursuing PHD from Jawaharlal Nehru Technological University, Hyderabad .His areas of interest includes WCDMA based Wireless Multimedia Networks and Cellular Mobile Communications .He is Presently working as an Associate Professor in ECE dept, Sreenidhi Institute of Science and Technology, Hyderabad Andhra Pradesh ,India.

Dr.S.VenkataChalam is graduated in B.Tech (Electronics and Communication Engineering) in 1987 from the University College JNTU College of Engineering Anantapur. He did his M.Tech in Microprocessor and application from M S University, Baroda during 1992.He was awarded Doctorate in ECE in 2004 from Jawaharlal Nehru Technological University, Hyderabad. His research area of interest includes Wireless Multimedia Networks, Embedded systems and Digital signal processing. He is presently guiding 10 research students of JNT University, Hyderabad. He has 33 publications in various National, International Conferences and Journals. Presently he is working as the principal of ACE Engineering College, Hyderabad, and Andhra Pradesh, India.

Dr.D.Sreenivasa Rao is graduated in B.Tech (Electronics and Communication Engineering) in 1986 from Nagarjuna University. He did his M.Tech in digital systems from Osmania University during 1994.He was awarded Doctorate in ECE in 2004 from University of Hyderabad .His research interest are in the area of communications and computer networks, Wireless Multimedia Networks. Presently 12 research students are working under his guidance. He has 23 publications in various National, International Conferences and journals.
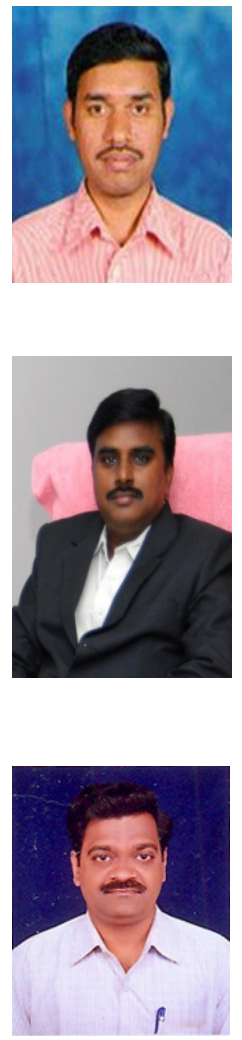